\title{
Study on Flow Characteristics in Volute of Centrifugal Pump Based on Dynamic Mode Decomposition
}

\author{
Yi-bin Li $(\mathbb{D}$, Chang-hong He $\mathbb{D}$, and Jian-zhong Li $(\mathbb{D}$ \\ College of Energy and Power Engineering, Lanzhou University of Technology, Lanzhou 730050, China \\ Correspondence should be addressed to Yi-bin Li; liyibin58@163.com
}

Received 31 January 2019; Accepted 4 April 2019; Published 16 April 2019

Academic Editor: Sergey A. Suslov

Copyright (C) 2019 Yi-bin Li et al. This is an open access article distributed under the Creative Commons Attribution License, which permits unrestricted use, distribution, and reproduction in any medium, provided the original work is properly cited.

\begin{abstract}
To investigate the unsteady flow characteristics and their influence mechanism in the volute of centrifugal pump, the Reynolds timeaveraged N-S equation, RNG $k-\varepsilon$ turbulence model, and structured grid technique are used to numerically analyze the transient flow-field characteristics inside the centrifugal pump volute. Based on the quantified parameters of flow field in the volute of centrifugal pump, the velocity mode contours and oscillation characteristics of the mid-span section of the volute of centrifugal pump are obtained by dynamic mode decomposition (DMD) for the nominal and low flow-rate condition. The research shows that the first-order average flow mode extracted by DMD is the dominant flow structure in the flow field of the volute. The secondorder and third-order modes are the most important oscillation modes causing unsteady flow in the volute, and the characteristic frequency of the two modes is consistent with the blade passing frequency and the $2 \mathrm{x}$ blade passing frequency obtained by the fast Fourier transform (FFT). By reconstructing the internal flow field of the volute with the blade passing frequency for the nominal flow-rate condition, the periodic variation of the unsteady flow structure in the volute under this frequency is visually reproduced, which provides some ideas for the study of the unsteady structure in the internal flow field of centrifugal pumps.
\end{abstract}

\section{Introduction}

The internal flow of the centrifugal pump is a complex threedimensional unsteady turbulent flow, often accompanied by flow separation, cavitation, hydraulic vibration, etc., which affect the stability of flow in the centrifugal pump. Among them, the rotor-stator interaction will produce periodic pressure fluctuation, which will lead to the intensification of pump vibration, noise enhancement, and performance decay, seriously affecting the safe and stable operation of the unit. Especially in the tongue area, since the fluid flow from the impeller outlet impacts the volute tongue, the rotorstator interaction at this place should be the strongest, which is one of the key areas for the study of unsteady flow in centrifugal pumps. For instance, Kelder [1] studied the flow in the volute of a low specific-speed pump both experimentally and numerically near its design point and found that near the design point of this pump, the core flow behaves like a potential flow, provided that no boundary layer separation occurs. González [2] captured the unsteady flow effects inside a centrifugal pump due to the impeller-volute interaction with a numerical simulation and provided the modeling possibilities for the prediction of the dynamic interaction between the flow at the impeller exit and the volute tongue. Keller [3] investigated the unsteady flow in a centrifugal pump with vaneless volute based on PIV measurements. The obtained data show that the fluid-dynamic blade-tongue interaction is dominated by high-vorticity sheets (positive and negative) being shed from the impeller channels, especially from the blade trailing edges, and their impingement on the tongue tip with subsequent. Tan [4] elucidated the detailed flow field and cavitation effect in the centrifugal pump volute at partial load condition. The results showed that the maximum amplitudes of pressure fluctuations in volute at serious cavitation condition are twice that at noncavitation condition because of the violent disturbances caused by cavitation shedding and explosion. Hamed [5] investigated reducing the radial force, the effects of concentric volute, and multivolute geometry on the head, efficiency, and radial force of a low speed centrifugal pump at off-design conditions. The results showed that the triple-volute is the most appropriate volute geometry at off-design conditions. 
Wang [6] carried out the transient analysis to investigate the dynamic stress and vibration of volute casing for a large double-suction centrifugal pump by using the transient fluid-structure interaction theory. The results revealed that, for all operating conditions, the maximum stress located at the volute tongue region, whereas the maximum vibration displacement happened close to the shaft hole region, and the blade passing frequency and its harmonics were dominant in the variations of dynamic stress and vibration displacement. Liu [7] investigated the pressure fluctuation intensity and vortex characteristic of a mixed-flow pump as turbine at pump mode with a tip clearance. Hao [8] studied the transient cavitating flows of a mixed-flow PAT (pump as turbine) at pump mode experimentally and numerically. Tan $[9,10]$ investigated the role of blade rotational angle in the energy performance and pressure fluctuation and the influence of original and T-shape blade end on performance of a mixed-flow pump through an experimental measurement and numerical simulation, respectively.

Dynamic mode decomposition (DMD) is a data-driven algorithm for extracting dynamic information from unsteady experimental measurements or numerical simulations. For such simulations and experiments provide large-scale data, it is necessary to understand essential phenomena from the data provided. So it can be used to analyze the main characteristics of complex unsteady flows or to establish low-order flow-field dynamics models. The DMD method was first proposed by Schmid [11-13] in 2008, and then the flow field data obtained by numerical simulation and experiment were analyzed by DMD method. Seena [14] employed the dynamic mode decomposition to analyze the unsteadiness in extracted modes without the explicit knowledge of evolution operator of the data. Hemati [15] presented two algorithms and compared the application of the two algorithms on cylinder wake data collected from both direct numerical simulations and PIV experiments. Tu [16] demonstrated the utility of this approach by presenting novel sampling strategies that increase computational efficiency and mitigated the effects of noise, respectively. Pan [17] used a linear combination of a sinusoidal unstable wave and its high-order harmonics as a prototype to take an error analysis of DMD algorithm. The result shows that the superimposition of finer structures with less energy dominance might damage the estimation accuracy of the primary structures' growth rate. Diana [18] proposed a new vector-filtering criterion for dynamic modes selection that is able to extract dynamically relevant flow features from dynamic mode decomposition of time-resolved experimental or numerical data. Kunihiko [19] described herein some of the dominant techniques for accomplishing these modal decompositions and analyses that have seen a surge of activity in recent decades and presented a brief overview of several of the well-established techniques and clearly lay the framework of these methods using familiar linear algebra. Liu [20, 21] employed the dynamic mode decomposition (DMD) and Proper Orthogonal Decomposition (POD) to analyze the coherent structure of cavitating flow around ALE 15 hydrofoil and investigated the complicated transient characteristics of gas-liquid two-phase flow in a multiphase pump under
TABLE 1: Main design parameters of the centrifugal pump.

\begin{tabular}{lc}
\hline Design parameter & Design value \\
\hline Nominal flow rate $Q_{\mathrm{d}}$ & $750 \mathrm{~m}^{3} / \mathrm{h}$ \\
Rated head $H_{\mathrm{d}}$ & $36 \mathrm{~m}$ \\
Rated rotational speed $n_{\mathrm{d}}$ & $1710 \mathrm{r} / \mathrm{min}$ \\
Number of blades $Z$ & 6 \\
Diameter of impeller inlet $D_{1}$ & $0.245 \mathrm{~m}$ \\
Diameter of impeller outlet $D_{2}$ & $0.325 \mathrm{~m}$ \\
Blade wrap angle $\alpha$ & $120^{\circ}$ \\
Blade outlet angle $\beta_{2}$ & $27^{\circ}$ \\
Base circle diameter of volute $D_{3}$ & $0.34 \mathrm{~m}$ \\
\hline
\end{tabular}

$10 \%$ and $20 \%$ inlet GVFs by dynamic mode decomposition (DMD).

\section{Numerical Model and Computational Method}

2.1. Geometry of the Pump. The object of this paper is a conventional single-stage single-suction centrifugal pump. The main parameters of the pump are listed in Table 1. The wrap angle $\alpha$ in Table 1 is defined as the angle between the line of the blade inlet edge and the center of the impeller and the line of the blade outlet edge and the center of the impeller. The three-dimensional water body (impeller and volute) required for the calculation of the model is generated by three-dimensional design software Pro/E 5.0. According to the design parameters, the number of blades is 6 , and the volute is a semihelical double volute. It should be pointed out that compared with a single volute, the presence of the baffle in the double volute divides the volute into two flow paths, and the volute tongue and the baffle entrance are symmetrical about the center of rotation. High-speed fluid from impeller passages still causes unsteady flow in volute when it impacts the inlet of baffle. Therefore, the existence of baffle is equivalent to the presence of a second tongue in double volute. So, this area also needs important attention in the study of internal flow in volute.

\subsection{Comparison of Numerical Simulation and Experiment in} the Centrifugal Pump. For the entire computational domain (composed of suction pipe, impeller, volute, and outlet pipe), structured hexahedral mesh is generated using Ansys-ICEM mesh generation tool. The overall calculation domain is shown in Figure 1. After the grid independence test, the total number of grids was 4.5 million. To calculate the unsteady flow structures in the centrifugal pump, the commercial CFD code Ansys-Fluent is used in the present paper. The RNG $k-\varepsilon$ two-equation model is used under the condition of satisfying the accuracy. The discrete method of the governing equation is the finite volume method. The coupling between the pressure and velocity is solved using the simple scheme, and second-order upwind scheme is used for spatial discretization. The uniform velocity at the pump inlet is set as the mass flow inlet boundary condition, and the outflow at the pump outlet is set as the outlet boundary condition. 


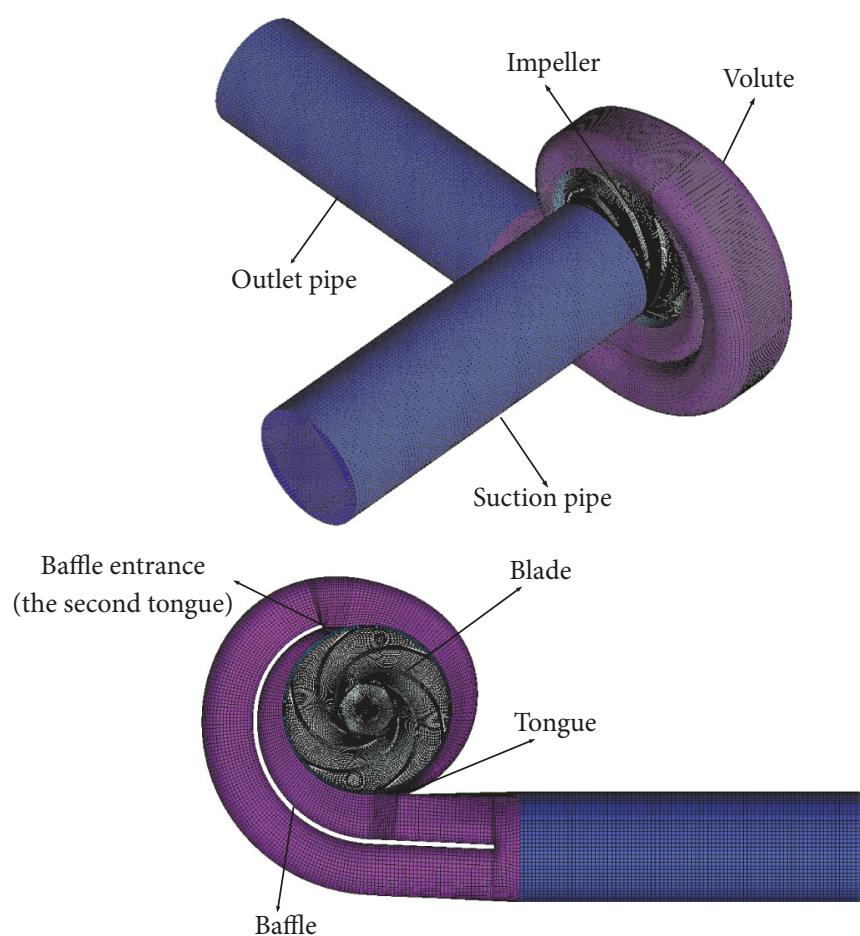

FIGURE 1: Structured grid of the centrifugal pump.

The fluid medium is the normal temperature water; and the boundary condition is the no-slip solid wall. To guarantee the accuracy of the result, the convergence precision of the continuity residual, $\mathrm{x}$-velocity residual, $\mathrm{y}$-velocity residual, $\mathrm{z}$ velocity residual, $\mathrm{k}$ residual, and epsilon residual for steady simulation is set as $10^{-5}$.

As shown in Figure 2, the performance test of the centrifugal pump was tested on a closed test stand. And Figure 3 shows the head/flow-rate relationship and the efficiency/flow-rate relationship curves obtained from both numerical simulation and experiment of the centrifugal pump. The results show that as the flow rate increases gradually, the head of the centrifugal pump gradually decreases, and the efficiency first increases and then decreases. For the different flow-rate conditions at the centrifugal pump, both the numerically calculated head and efficiency values are higher than the experimental values, and the errors for the nominal flow-rate condition are $5.8 \%$ and $3.7 \%$, respectively. The errors between numerical simulation and experiment are due to the fact that the mechanical losses caused by bearings, mechanical seals, etc. are often ignored in numerical calculation. From the comparison of numerical simulation and experimental values of head and efficiency of the centrifugal pump, the numerical simulation method was used to predict the performance of the centrifugal pump having good accuracy of the reference value. In addition, it can be seen from Figure 3 that in the efficiency/flow-rate curve and head/flow-rate curve, the maximum difference appears at the nominal flow-rate condition, but the values obtained by numerical calculation are in good agreement with the experimental values for the low flow-rate condition.
Therefore, it can be explained that the centrifugal pump is in a state of off-design condition when it works. The main reason for this phenomenon is that the design parameters of impeller are unreasonable, such as the impeller outlet diameter and the impeller outlet width being too small.

\section{Dynamic Mode Decomposition}

Dynamic mode decomposition is a method for extracting the mode in a flow based on flow-field snapshots, so that the flow structure can be accurately described. For the linear flow, the DMD method can extract the modes that can characterize the global flow stability. For the nonlinear flow, the DMD method can describe the flow structure in which the observations (such as velocity and pressure) dominate. The mathematical derivation process of it is introduced below.

Suppose there is a set of observation data matrices that vary with time:

$$
V_{1}^{\mathrm{N}}=\left[v_{1}, v_{2}, \cdots, v_{N-1}, v_{N}\right]
$$

where $\mathrm{N}$ is the total number of snapshots of the flow field, and the column vector $\boldsymbol{v}_{\boldsymbol{i}}$ is the data of the $i$ th snapshot. It is assumed that the snapshot data of the flow field at two adjacent moments can be represented by a linear transformation matrix $A$ :

$$
v_{i+1}=A v_{i}
$$

As the acquired snapshot data increases, we can further assume that the vector formed by the snapshot data eventually 

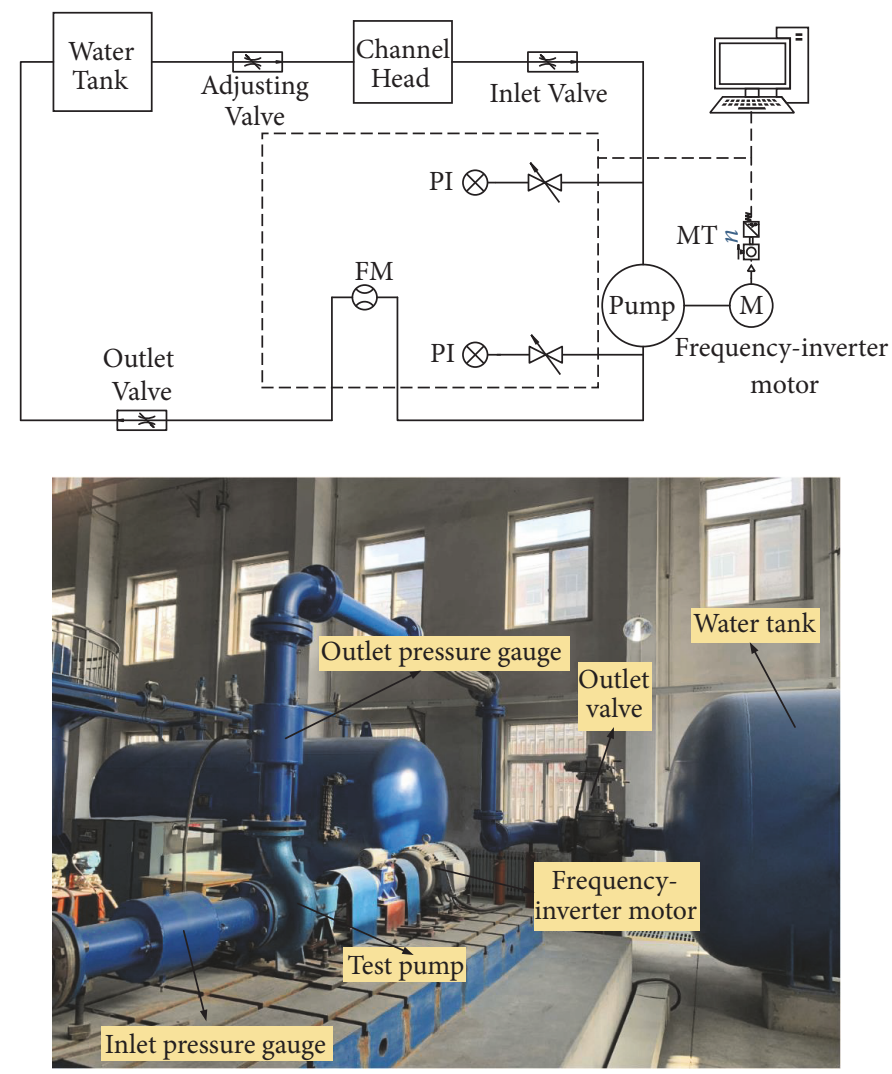

Figure 2: Test stand.

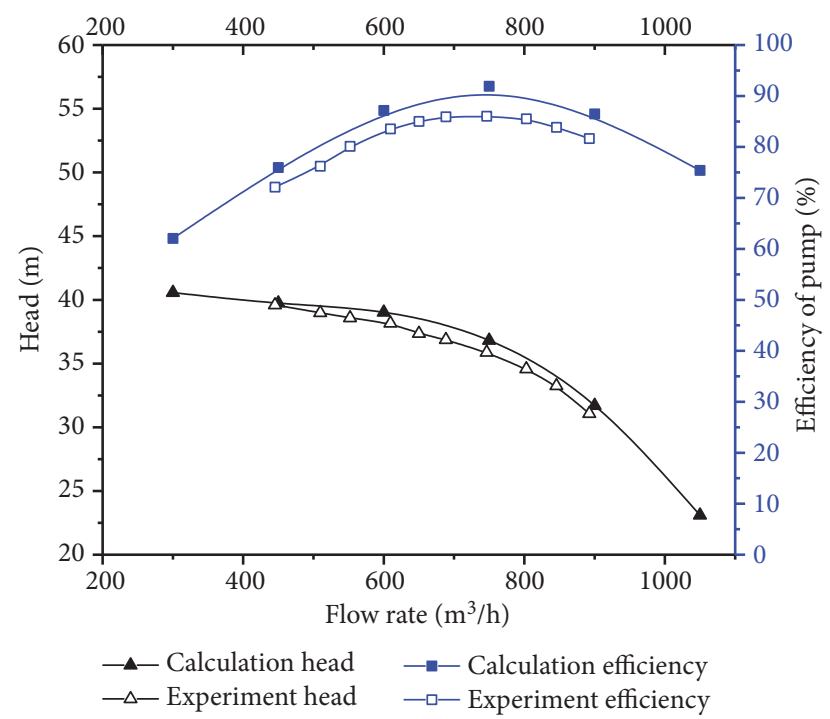

FIGURE 3: External characteristic curve of the centrifugal pump.

tends to be linearized. Thus, the last flow-field snapshot can be represented as a linear combination of all previous snapshots:

$$
v_{N}=a_{1} v_{1}+a_{2} v_{2}+\cdots+a_{N-1} v_{N-1}+r
$$

The matrix form is

$$
v_{N}=V_{1}^{N-1} a+r
$$

In formula (4), $a^{T}=\left[a_{1}, a_{2}, \cdots, a_{N-1}\right] ; r$ is residual vector.

From formula (1),

$$
V_{2}^{N}=\left[A v_{1}, A v_{2}, \cdots, A v_{N-1}\right]=A V_{1}^{N-1}
$$

The new relation can be obtained by combining formula (3) as follows:

$$
A V_{1}^{N-1}=V_{2}^{N}=V_{1}^{N-1} S+r e_{N-1}^{T}
$$

In formula (6),

$$
S=\left(\begin{array}{ccccc}
0 & \cdots & 0 & 0 & a_{1} \\
1 & \ddots & \vdots & \vdots & \vdots \\
0 & \ddots & 0 & 0 & a_{N-3} \\
\vdots & \ddots & 1 & 0 & a_{N-2} \\
0 & \cdots & 0 & 1 & a_{N-1}
\end{array}\right)
$$

Obviously, when the residual vector $r$ is small, the eigenvalue of the matrix $S$ is approximated by the eigenvalue of the system matrix $A$. Therefore, the matrix $S$ is a low-dimensional approximation of the system matrix $A$, and its eigenvalues can 
represent the main eigenvalues of the system matrix $A$. Matrix $S$ is usually obtained by $Q R$ decomposition of matrix $V_{1}^{N-1}$; if matrix $V_{1}^{N-1}$ can not guarantee full rank, its QR decomposition is not unique and may lead to the irreversibility of upper triangular matrix $R$, which will ultimately lead to the impossibility of finding matrix $S$. Perform singular value decomposition on matrix $V_{1}^{N-1}$ :

$$
V_{1}^{N-1}=U \Sigma V^{H}
$$

From formula (5) and formula (7):

$$
U^{H} A U=U^{H} A\left(U \Sigma V^{H}\right) V \Sigma^{-1}=U^{H} V_{2}^{N} V \Sigma^{-1} \equiv \widetilde{S}
$$

From the previous analysis, we can see that the eigenvalues of matrix $\widetilde{S}$ can represent the main eigenvalues of system matrix $\boldsymbol{A}$. Then the eigenvalues and eigenvectors of matrix $\widetilde{S}$ are obtained:

$$
\widetilde{S} \mu_{i}=w_{i} \mu_{i}
$$

Finally, the DMD modes can be obtained as follows:

$$
\Phi_{i}=U w_{i}
$$

It should be pointed out that the eigenvalue $\mu_{i}$ contains the information of the mode $\Phi_{i}$. When the eigenvalues are expressed in the complex plane, the modes on the unit circle of the complex plane are relatively steady, while the modes whose eigenvalues are not on the unit circle are unsteady.

The corresponding frequency $\omega_{i}$ and growth rate $\varphi_{i}$ of the modes are defined as follows.

$$
\begin{aligned}
\omega_{i} & =\frac{\operatorname{Im}\left(\ln \left(\mu_{i}\right) / \Delta t\right)}{2 \pi} \\
\varphi_{i} & =\operatorname{Re}\left(\frac{\ln \left(\mu_{i}\right)}{\Delta t}\right)
\end{aligned}
$$

According to the similar matrix $\widetilde{S}$ obtained by the above DMD decomposition, the evolution of the flow field can be further estimated. By singular value decomposition (7), a high-dimensional system $\boldsymbol{v}_{\boldsymbol{i}}$ can be mapped to a subspace $z_{\boldsymbol{i}}$.

$$
z_{i}=U^{H} v_{i}
$$

By simple transformation there are

$$
z_{i+1}=U^{H} v_{i+1}=U^{H} A v_{i}=U^{H} A U z_{i}=\widetilde{S} z_{i}
$$

Let $w_{i}$ be the column vector of matrix $W$; $N$ has the eigenvalues of the matrix $\widetilde{S}$ in its diagonal. Then the eigendecomposition can be expressed as

$$
\widetilde{S}=W N W^{-1}
$$

So, from the previous derivation, a snapshot at any time instant $i$ can be approximated as

$$
\begin{aligned}
v_{i} & =A v_{i-1}=U \widetilde{S} U^{H} v_{i-1}=U W N W^{-1} U^{H} v_{i-1} \\
& =U W N^{i-1} W^{-1} U^{H} v_{1}
\end{aligned}
$$

From the DMD mode definition formula (10),

$$
\Phi=U W
$$

The mode amplitude $\alpha$ is represented as

$$
\alpha=W^{-1} z_{1}=W^{-1} U^{H} v_{1}
$$

where $\alpha_{i}$ denotes the amplitude of the $i$ th mode, which represents the mode contribution to the initial snapshot $v_{1}$.

Substituting formula (17) and (18) into formula (16), the flow field at any time instant can be predicted as

$$
v_{i}=\sum_{j=1}^{r} \Phi_{j}\left(\mu_{j}\right)^{i-1} \alpha_{j}
$$

Then its snapshot sequences can be expressed as

$$
\begin{gathered}
{\left[v_{1}, v_{2}, \cdots, v_{N-1}\right]=\left[\Phi_{1}, \Phi_{2}, \cdots, \Phi_{r}\right]} \\
\cdot\left[\begin{array}{ccccc}
\alpha_{1} & & & 0 \\
& \alpha_{2} & & \\
& & \ddots & \\
& & & \alpha_{r}
\end{array}\right]\left[\begin{array}{cccc}
1 & \mu_{1} & \cdots & \mu_{1}^{N-1} \\
1 & \mu_{2} & \cdots & \mu_{2}^{N-1} \\
\vdots & \vdots & \ddots & \vdots \\
1 & \mu_{r} & \cdots & \mu_{r}^{N-1}
\end{array}\right]
\end{gathered}
$$

The dynamic mode decomposition of the velocity field in the mid-span section of the centrifugal pump volute can be performed by using the DMD calculation formula derived above. Firstly, we need to edit the formula of DMD calculation in MATLAB and then export the coordinates of grid points in the mid-span section and the velocity values at the grid points in CFD Post. From the definition above, it can be seen that the set of velocity values at different grid points at the same time is a column vector of the whole data matrix. The final calculation data matrix is made up of the set of velocity values at different times. Next, the dynamic mode decomposition of the velocity matrix is then performed using MATLAB. Finally, the calculated modal values are edited into the acceptable data format of CFD Post and imported into CFD Post to make the corresponding velocity contours.

\section{Results and Discussions}

4.1. Unsteady Pressure Fluctuation Analysis inside the Volute. In the unsteady calculation, the spectrum analysis is performed to obtain the characteristic frequency of the unsteady flow inside the centrifugal pump. The dimensionless pressure coefficient $C_{p}$ is introduced to describe the pressure fluctuation characteristics of each monitoring point. The expression is as follows:

$$
C_{p}=\frac{\left(P_{i}-P_{a v e}\right)}{(1 / 2) \rho u_{2}^{2}}
$$

where $P_{i}$ is the static pressure value of the monitoring point at a certain time, $\mathrm{P}_{\mathrm{a}} ; P_{\text {ave }}$ is the average value of static pressure in one cycle, $\mathrm{P}_{\mathrm{a}} ; u_{2}$ is the circumferential velocity 


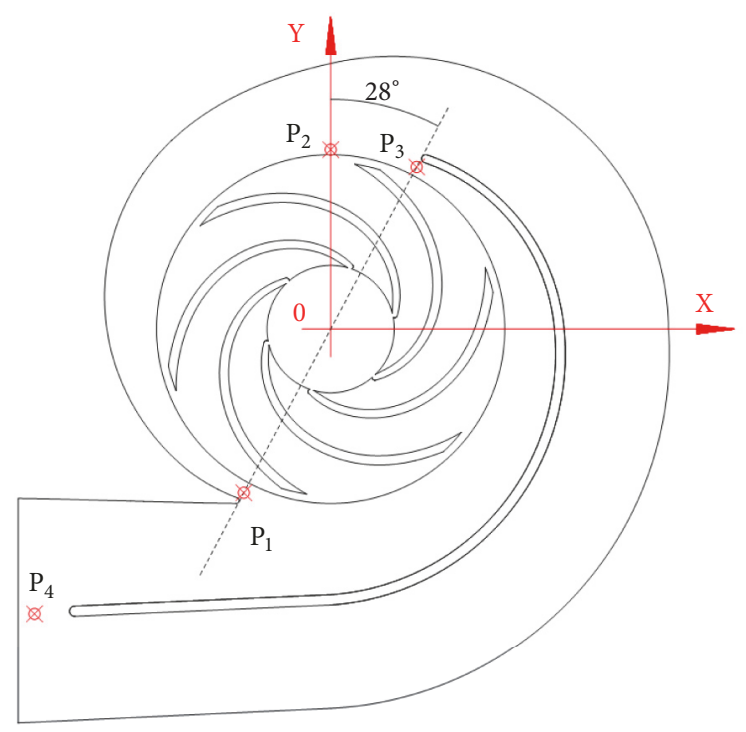

FIGURE 4: Locations of monitoring points.

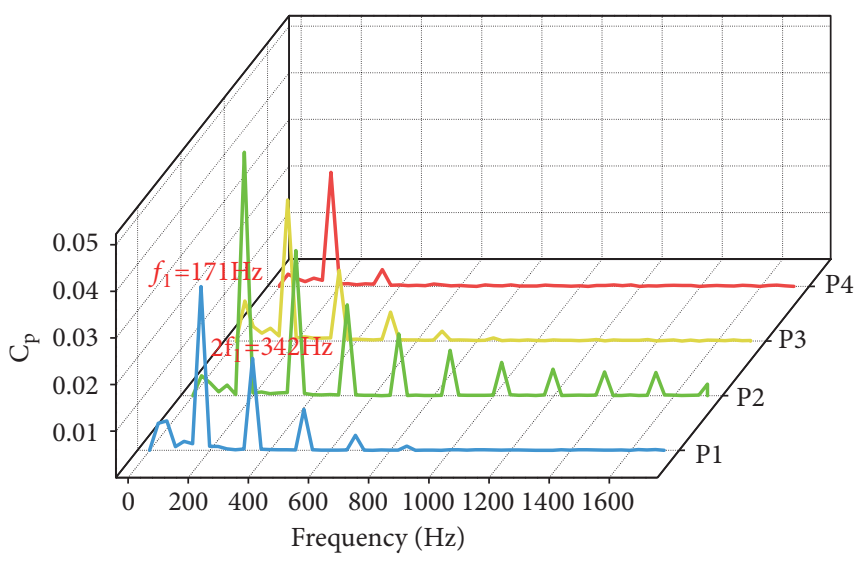

FIGURE 5: Spectrum characteristic analysis for the low flow-rate condition.

at the impeller outlet, $\mathrm{m} / \mathrm{s}$. The monitoring points $P_{1}, P_{2}, P_{3}$, and $P_{4}$ are arranged inside the volute as shown in Figure 4. $P_{1}$ and $P_{3}$ are set to monitor pressure fluctuation characteristics of fluid flow in volute tongue and baffle entrance region; $P_{2}$ is set to monitor the pressure fluctuation characteristics of fluid flow at the volute inlet except for the tongue and the baffle entrance region; and $\mathrm{P} 4$ is set to monitor the pressure fluctuation characteristics at the volute outlet. By comparing the pressure fluctuation characteristics at these four points, the main factors causing unsteady flow in volute are explored.

Figure 5 shows the spectrum analysis results of the pressure fluctuation at the monitoring points inside the centrifugal pump for the low flow-rate $\left(0.5 Q_{\mathrm{d}}\right)$ condition. Obviously, the main frequency of the pressure fluctuation in the centrifugal pump is the blade passing frequency $\left(f_{1}=171 \mathrm{~Hz}\right)$, and the pressure amplitude at $2 x$ blade passing frequency $\left(2 f_{1}=342 \mathrm{HZ}\right)$ is also prominent. Figure 6 shows the spectrum analysis results of the pressure fluctuation at the monitoring points inside the centrifugal pump under the low flow-rate $\left(0.5 Q_{d}\right)$ condition. For the nominal flowrate condition, the blade passing frequency of the centrifugal pump is $f_{1}=169.59 \mathrm{~Hz}$ and the $2 x$ blade passing frequency is $2 f_{1}=339.17 \mathrm{HZ}$. Comparing the results of spectrum analysis for the two operating conditions, it can be seen that the dominant pressure fluctuation main frequency and the high amplitude pressure fluctuation frequency are basically consistent. However, compared with the nominal flow-rate condition, it produces a certain low frequency fluctuation at less than the blade passing frequency in the low flow-rate condition.

4.2. Modal Analysis inside the Volute at the Low Flow-Rate Condition. Based on the previous CFD unsteady calculation results, DMD analysis was performed on 120 flow-field speed snapshots with time interval $\Delta t=0.0015 \mathrm{~s}$, and the DMD results for the two operating conditions were obtained. 


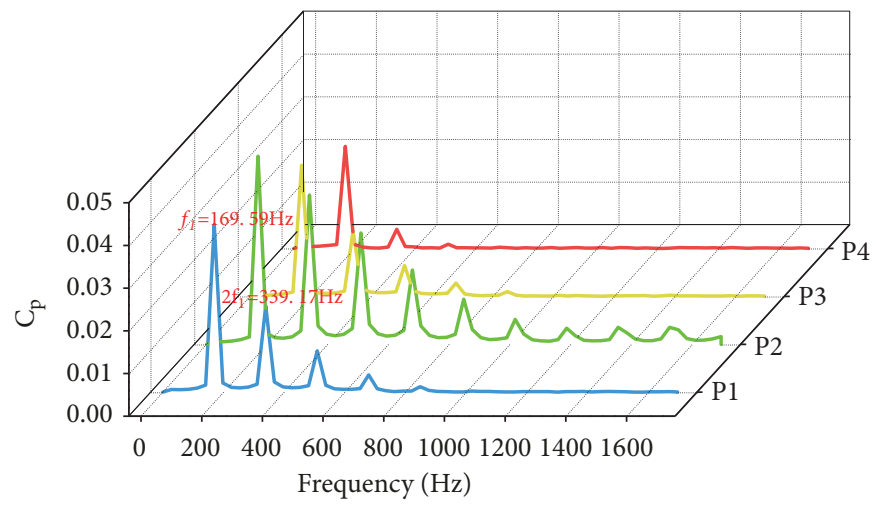

FIGURE 6: Spectrum characteristic analysis for the nominal flow-rate condition.

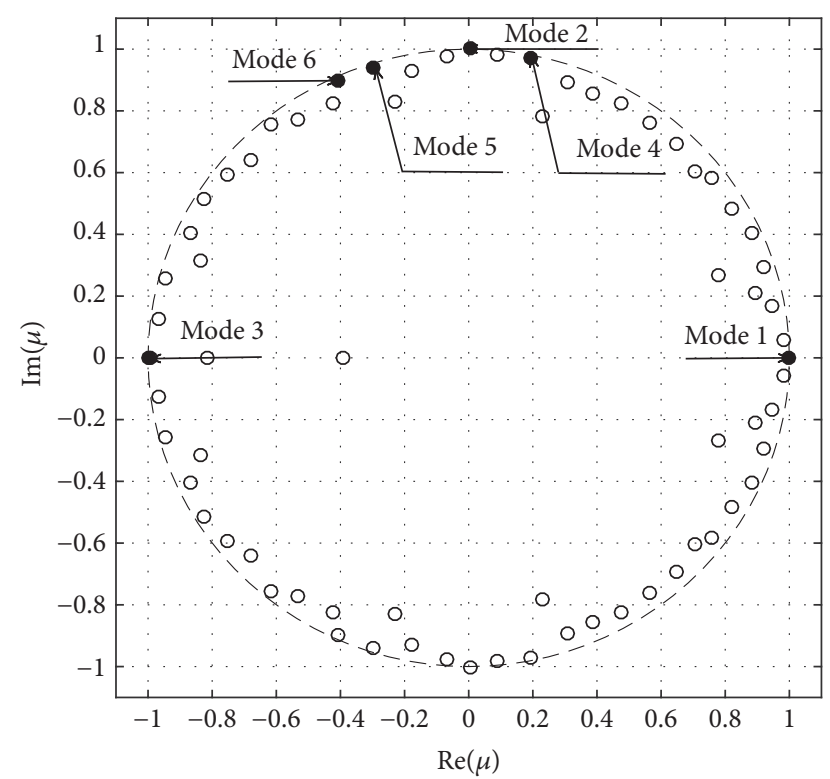

FIGURE 7: Distribution of DMD eigenvalues for the low flow-rate condition.

Figure 7 shows the distribution of the DMD mode eigenvalue $\mu_{\mathrm{i}}$ in the complex plane of the unsteady flow field in the midspan section of the volute of the centrifugal pump at low flow-rate $\left(0.5 Q_{d}\right)$ condition. In this figure, the real part of the eigenvalue is the horizontal axis and the imaginary part is the vertical axis. All the eigenvalues are distributed near the unit circle, and some eigenvalues are distributed on the unit circle, which means the corresponding modes are neutral stability. Figure 8 shows the relationship between the corresponding frequency of the DMD modes and the correlation coefficient. The correlation coefficient is used to measure the influence of each mode on the original flow field. In [22], $C_{j}$ is defined as the correlation coefficient.

$$
C_{j}=\sum_{i=1}^{\mathrm{N}}\left|\alpha_{j}\left(\mu_{j}\right)^{i-1}\right|\left\|\Phi_{j}\right\|_{F}^{2} \times \Delta t
$$

where $\alpha_{j}$ is the modal amplitude and $\mu_{j}$ is the eigenvalue and $\left\|\Phi_{j}\right\|_{F}^{2}$ is the mode Frobenius norm. After sorting the DMD modes according to the correlation coefficient, the first six modes with the highest correlation coefficient can be clearly seen from Figure 8 . Since the corresponding eigenvalue of the mode with the largest correlation coefficient is a real number, its frequency is zero. Other eigenvalues are complex numbers, and conjugate pairs appear, which can be observed from the distribution of eigenvalues. Since the parameters describing the flow-field information in each mode are their corresponding real parts, modes corresponding to a pair of conjugate eigenvalues are the same mode. The frequencies of the second and third-order modes are consistent with the blade passing frequency and $2 x$ blade passing frequency of the centrifugal pump obtained by the FFT in Figure 5, which fully demonstrates that the pressure fluctuation frequency in the original flow field is objectively present, and the capture of the characteristic frequency by the DMD is very accurate. From Figures 7 and 8, it can be clearly seen that the highenergy mode eigenvalues are distributed on the unit circle, so the corresponding flow is relatively steady, which belongs to the main flow structure in the flow field, while the flow corresponding to the low-energy mode eigenvalues which are not distributed on the unit circle is unsteady, and it is not the main flow structure in the flow field.

Figure 9 is the first sixth-order mode velocity contour of the mid-span section of the volute for the low flowrate condition $\left(0.5 Q_{d}\right)$. Figure $9(a)$ is the first-order zerofrequency velocity mode, and the eigenvalue corresponding to this mode is real number, so the frequency of this mode is zero; that is, the flow structure is time-average and is the average flow mode of the velocity field, which shows the dominant flow structure in the original flow field. Actually, the average flow mode can be regarded as the basic structure of the velocity field inside the volute, and the original flow field inside the volute can be regarded as being formed by superimposing oscillation modes of different frequencies on this basic structure. It can be seen from the velocity contour that due to the existence of the volute tongue and the volute baffle, two high-speed fluid regions are distributed at the entrance of the tongue and the baffle, which affects the circumferential distribution of the speed in the volute inlet. Due to the existence of the baffle, the velocity field of the basic flow structure inside the volute is symmetrically distributed 


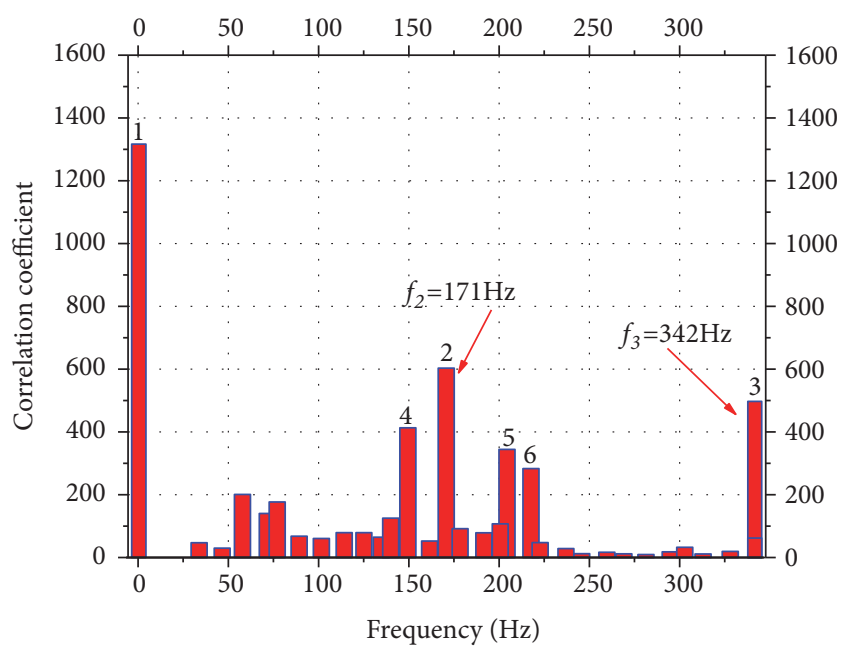

Figure 8: Distribution of correlation coefficients for the low flowrate condition.

with respect to the center of rotation, which also changes the flow state inside the volute to some extent.

Figures 9(b) and 9(c) are velocity contour of the secondorder and third-order modes of the mid-span section of the volute for the low flow-rate condition $\left(0.5 Q_{d}\right)$. From the comparison of correlation coefficients, the second- and thirdorder velocity modes are the two most important modes besides the first-order zero-frequency modes. Therefore, the two-order velocity modes are also the main oscillating modes causing unsteady flow in the volute. From the velocity contour in the second- and third-order modes, it can be seen that there are 6 and 12 periodic high and low velocity clusters in the circumference of the volute inlet, respectively. However, due to the influence of the tongue and the baffle, the high and low velocity fluid regions alternately distributed in the two places are weakened to a certain extent. The frequencies of the two-order velocity modes correspond exactly to the blade passing frequency and $2 x$ blade passing frequency obtained in the FFT, which indicates that the DMD captures the influence of the centrifugal pump rotor-stator interaction flow structure. Figures 9(d), 9(e), and 9(f) are the velocity contour of the fourth, fifth, and sixth modes of the mid-span section of the volute, respectively. The fourth-order mode captures the unstable flow structure caused by three high velocity fluid clusters downstream of the baffle inlet. It can be seen from the fifth order mode velocity contour that high and low velocity fluid clusters alternately distribute at the inlet of the tongue and the baffle. And high and low velocity fluid clusters alternately distribute in a direction perpendicular to the line connecting the tongue and the partition in the sixth order mode velocity contour. The distribution indicates that there are vortex structures with opposite rotation directions, reflecting the unsteady characteristics of high-order flow inside the volute.

4.3. Modal Analysis inside the Volute at the Nominal FlowRate Condition. Figures 10 and 11 show the distribution of the DMD mode eigenvalues and correlation coefficients of the unsteady flow field in the mid-span section of the volute of the centrifugal pump for the nominal flow-rate condition $\left(Q_{\mathrm{d}}\right)$. In the distribution of eigenvalues, it can be seen that the eigenvalues of the two modes are in the neutral stable range of the unit circle, but because of their low correlation coefficients, the influence on the original flow field is weak.

Figure 12 is the first six-order velocity mode contour of the mid-span section of the volute for the nominal flowrate condition $\left(Q_{d}\right)$, where Figure 12(a) is still the first-order zero-frequency mode, which is also the basic structure of the velocity field inside the volute. The velocity contour of the first-order mode is similar to the zero-frequency velocity contour in the low flow-rate condition. A high-speed fluid region is evenly distributed in the circumferential direction at the volute inlet, and the flow of fluid is still symmetrical about the center of the axis of rotation in the two channels of the volute. The basic flow structure in the volute is still affected by the presence of the tongue and the baffle, but the degree of influence is greatly reduced compared to the low flow-rate condition.

Figures 12(b) and 12(c) are the second- and third-order velocity mode contour of the mid-span section of the volute for the nominal flow-rate condition $\left(Q_{d}\right)$, which are similar to the corresponding modes for the low flow-rate condition. The two modes are still the main oscillation mode for causing unsteady flow in the volute. Similarly, the frequencies of the two modes are the same as the blade passing frequency and $2 x$ blade passing frequency of the centrifugal pump, which indicates that whether it is in a low or nominal flow-rate condition, the rotor-stator interaction of the impeller and the volute is the main cause of the unsteady flow in the volute of the centrifugal pump. However, unlike the low flow-rate condition, the periodic velocity clusters in the two velocity modes are almost not affected by the tongue and baffle for the nominal flow-rate condition.

Figures 12(d), 12(e), and 12(f) are the fourth-, fifth-, and sixth-order velocity mode contour of the mid-span section of the volute for the nominal flow-rate condition $\left(Q_{d}\right)$. In these order velocity mode contours, there are 8,10 , and 7 periodic velocity clusters in the inlet of the volute, respectively. It can be seen from the velocity contours that the influence range of the high and low velocity fluid clusters in these three modes on the flow field is much smaller than that in the secondand third-order modes in the volute, which is also consistent with the distribution of correlation coefficients. In addition, compared with similar modes for low flow-rate condition, the distribution of unsteady fluid clusters is still not affected by the tongue and baffle in the latter three modes for the nominal flow-rate condition. Combining with the second and third-order modes, it shows that the existence of the tongue and baffle can restrain the unsteady flow structure inside the volute when the flow rate of the centrifugal pump decreases. It should also be pointed out that except the second- and third-order modes which cause unsteady flow in the volute, the other three modes are all distributed between the blade passing frequency and $2 x$ blade passing frequency for the nominal flow-rate condition, while the frequency of the fourth-order mode with a high correlation coefficient is less than the blade passing frequency for the low flow-rate 


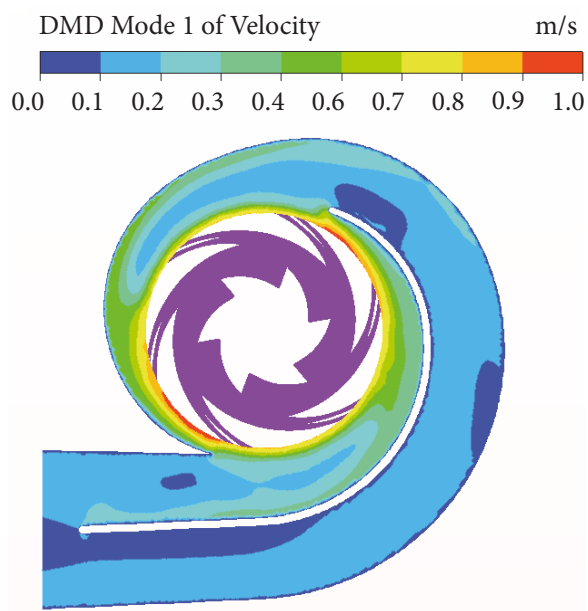

(a) First-order mode
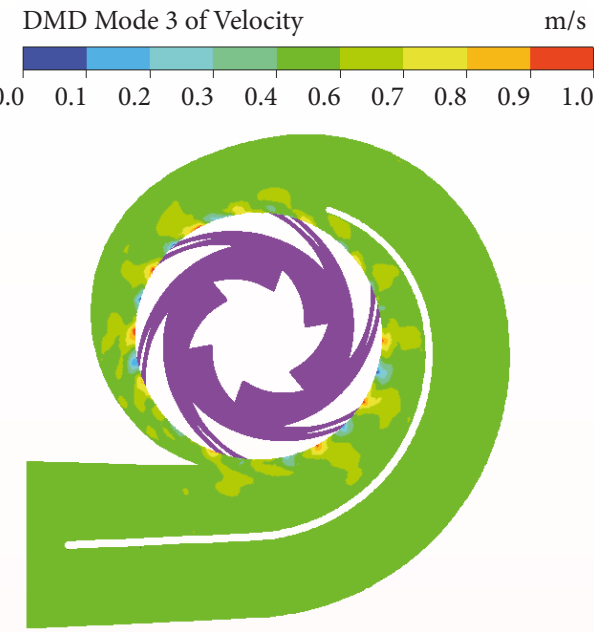

(c) Third-order mode

DMD Mode 5 of Velocity

$\mathrm{m} / \mathrm{s}$
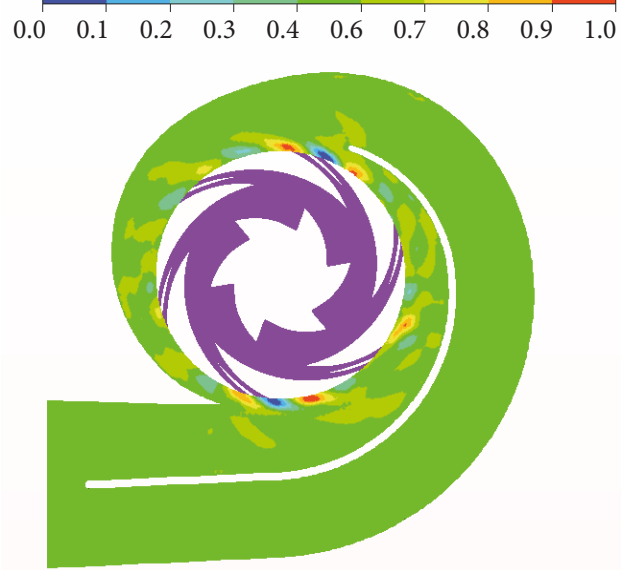

(e) Fifth-order mode
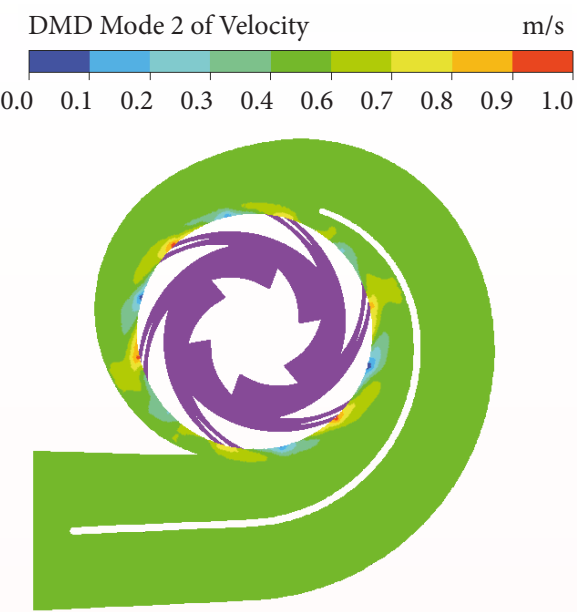

(b) Second-order mode

DMD Mode 4 of Velocity
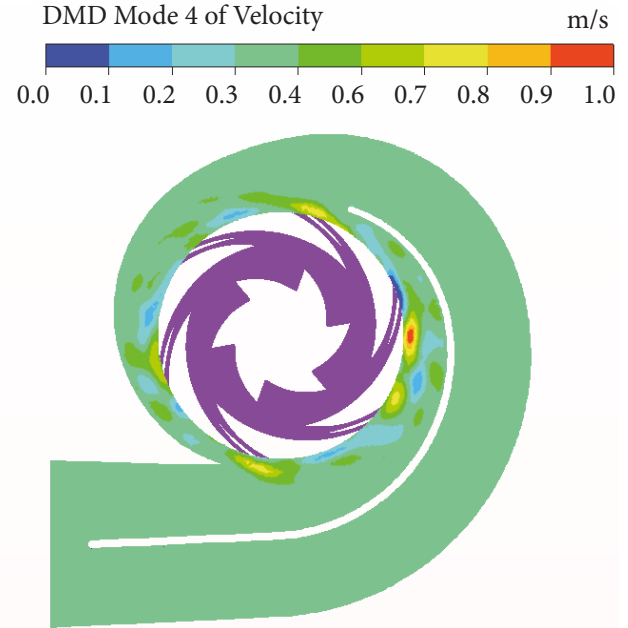

(d) Fourth-order mode

DMD Mode 6 of Velocity $\mathrm{m} / \mathrm{s}$

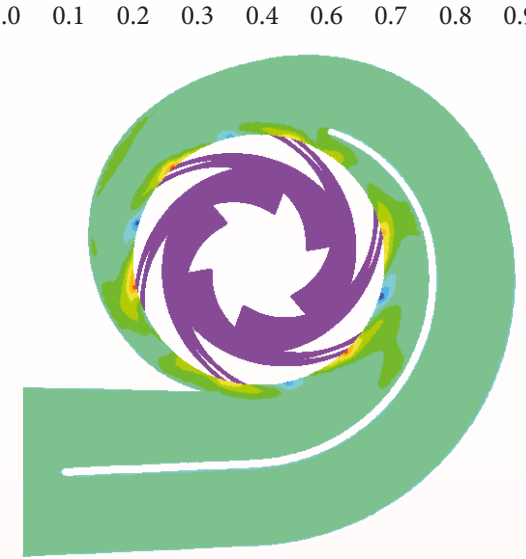

(f) Sixth-order mode

FIGURE 9: DMD velocity mode contours for the low flow-rate condition. 


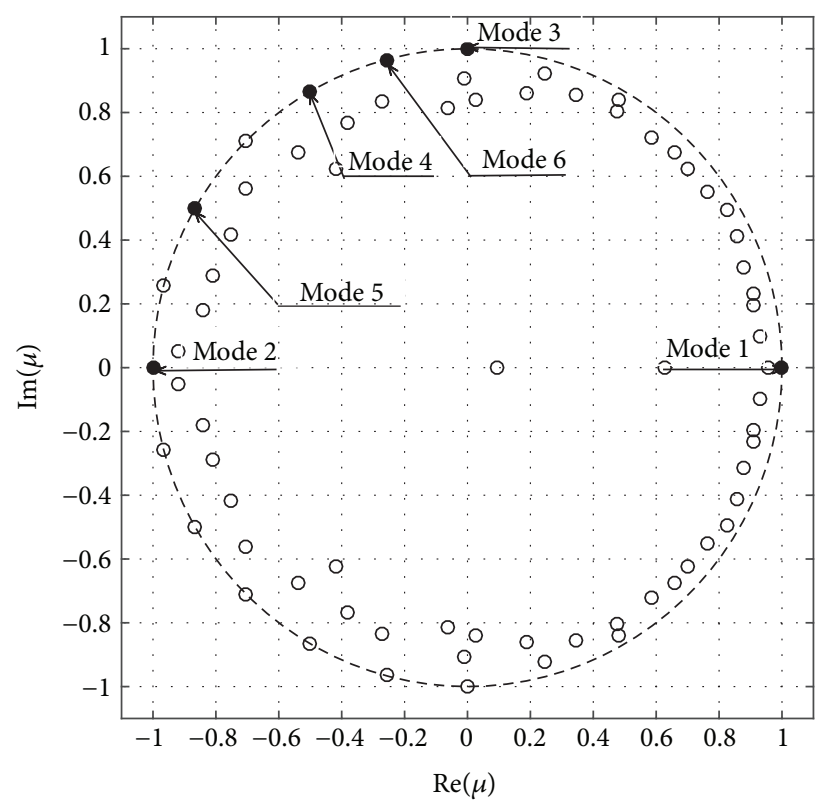

FIgURE 10: Distribution of DMD eigenvalues for the nominal flow-rate condition.

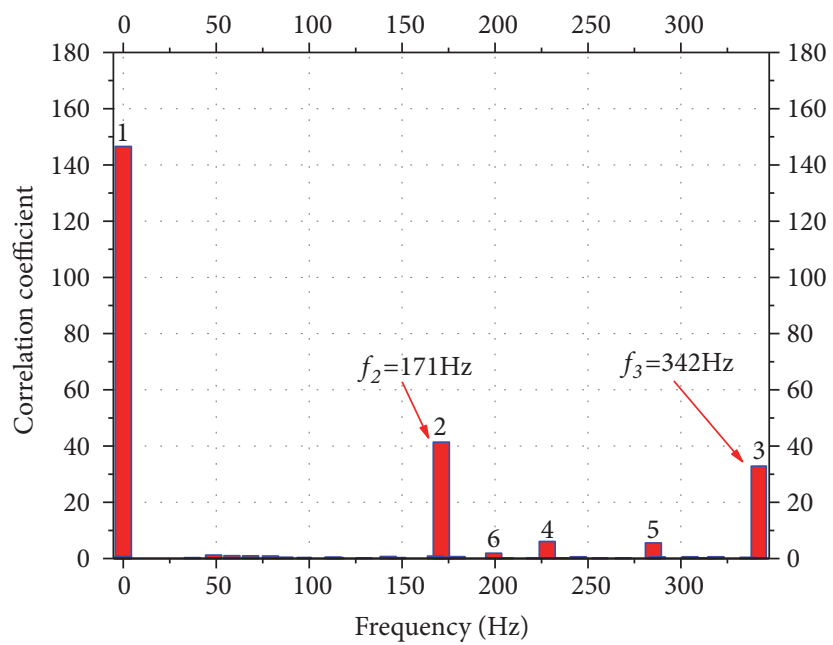

FIGURE 11: Distribution of correlation coefficients for the nominal flow-rate condition.

condition $\left(0.5 Q_{\mathrm{d}}\right)$, which indicates that the unsteady flow in the volute is more prone to occur at low frequencies when the flow rate decreases.

\subsection{Reconstruction of Flow Field in Volute Based on DMD.} In order to further observe the effect of dynamic mode decomposition on the extraction of flow-field characteristics in volute of centrifugal pump, a reduced order model of unsteady flow field in volute was established based on formula (19), and the flow field was reconstructed by using the obtained dynamic mode decomposition method. The unsteady flow field is reconstructed by the first ten modes with the highest correlation coefficient obtained by DMD. Figures 13 and 14 show the comparison of the reconstructed velocity contour and the original flow- field velocity contour at $\mathrm{T} / 2$ for the low flow-rate condition and the nominal flowrate condition. From the comparison, it can be seen that the reconstructed results have a high degree of reduction and identification for the flow structure in the flow field.

Since the first-order mode is an average flow mode, it does not change during the entire rotating period of the centrifugal pump. In order to further study the unsteady flow structure in a certain mode, the mode can be superimposed on the first-order average flow mode to observe the oscillation law of the mode. The second-order mode caused by rotor-stator interaction is superimposed on the average flow mode to reconstruct the flow field of a single mode, so as to observe the variation of the unsteady structure with time in the mode.

Figure 15 shows the reconstructed velocity contour of the mode corresponding to the blade passing frequency at 


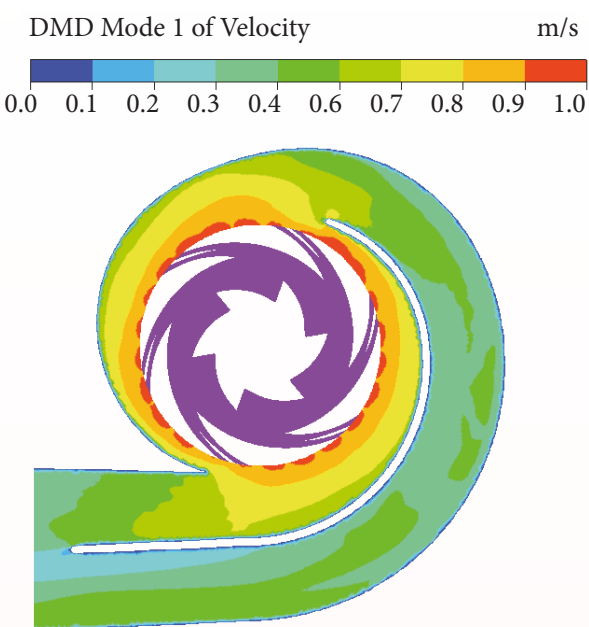

(a) First-order mode

\section{DMD Mode 3 of Velocity}
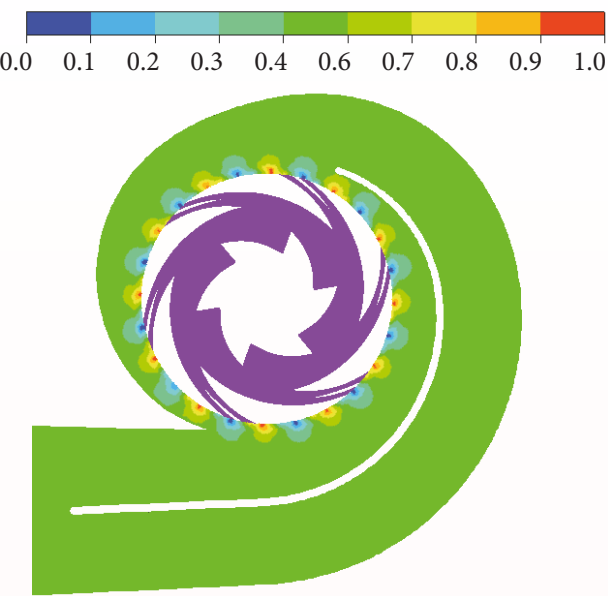

(c) Third-order mode

\section{DMD Mode 5 of Velocity}
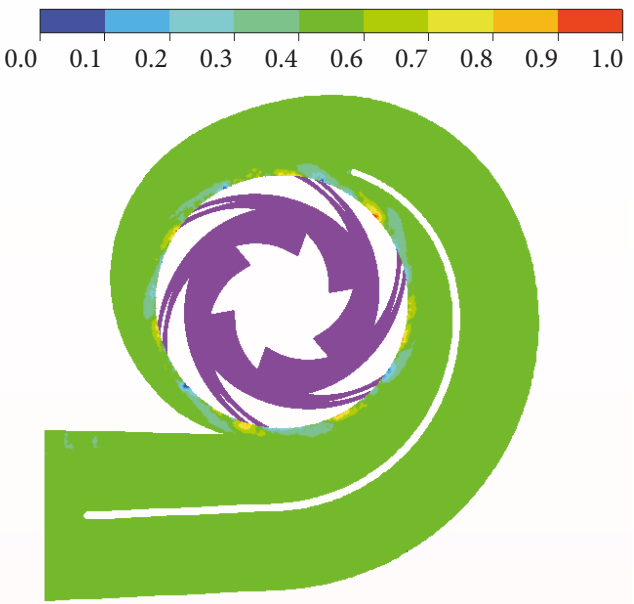

(e) Fifth order mode

\section{DMD Mode 2 of Velocity \\ $\mathrm{m} / \mathrm{s}$}

$\begin{array}{llllllllll}0.0 & 0.1 & 0.2 & 0.3 & 0.4 & 0.6 & 0.7 & 0.8 & 0.9 & 1.0\end{array}$

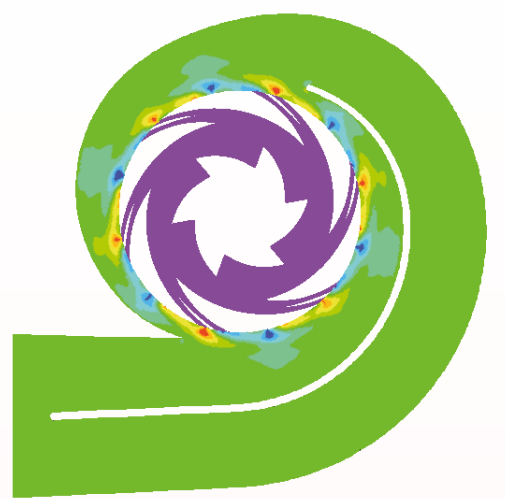

(b) Second-order mode
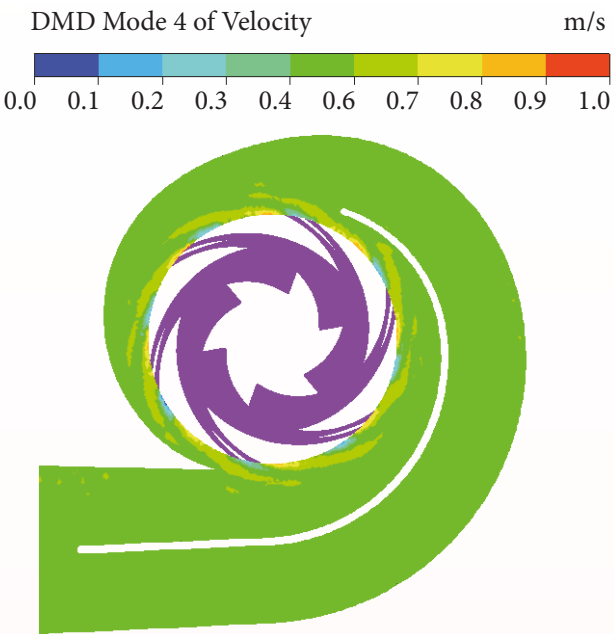

(d) Fourth order mode

DMD Mode 6 of Velocity

$\mathrm{m} / \mathrm{s}$
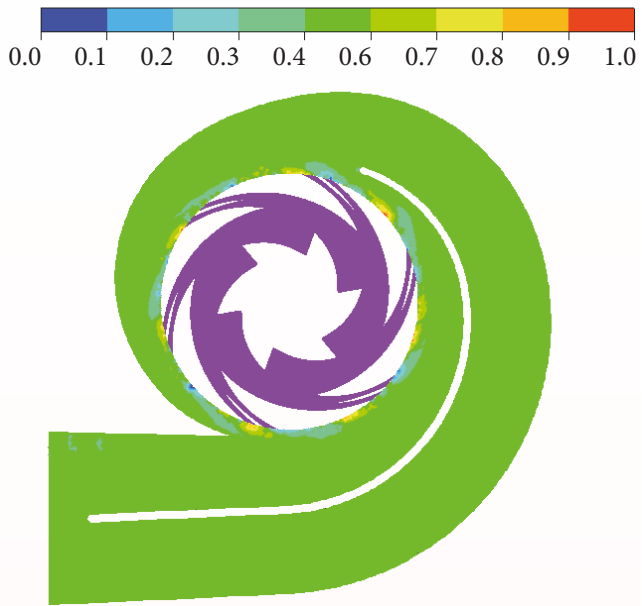

(f) Sixth order mode

FIGURE 12: DMD velocity mode contours for the nominal flow-rate condition. 

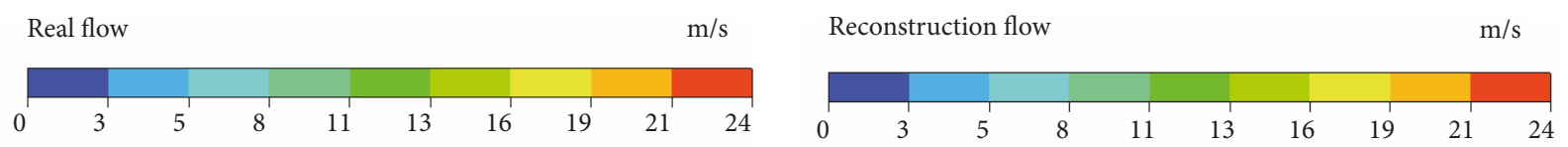

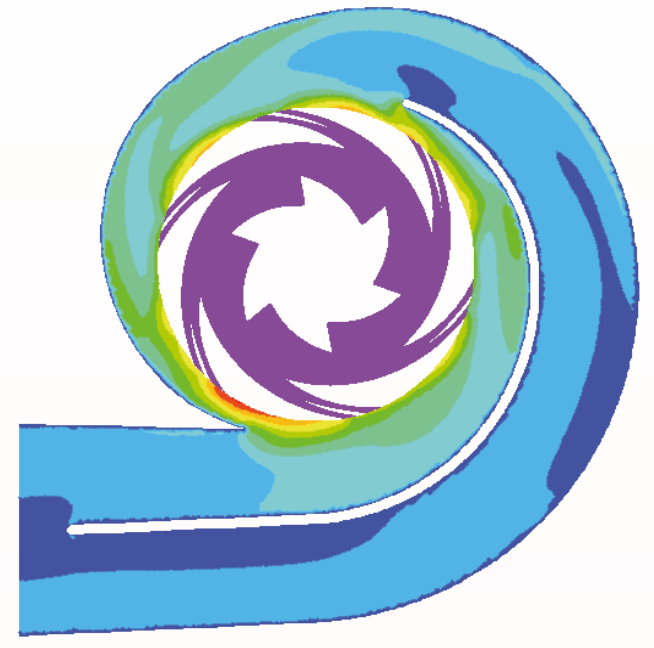

(a) Original flow field at $\mathrm{T} / 2$

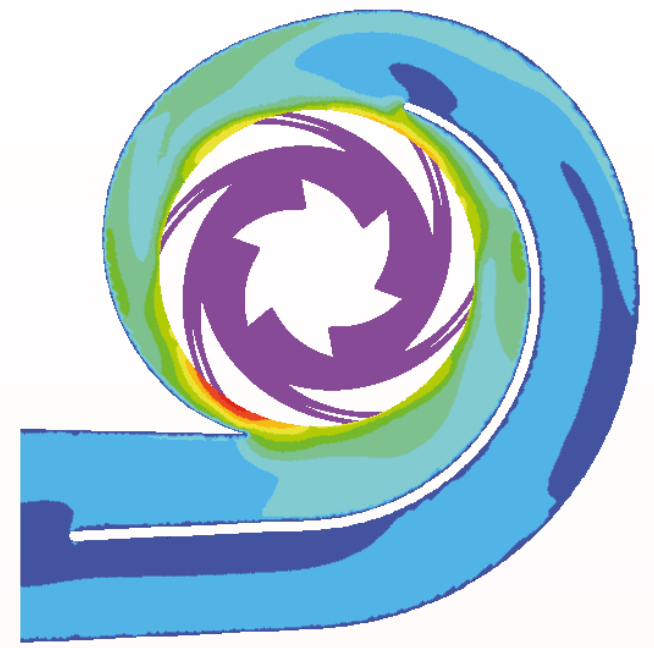

(b) Reconstructed flow field at T/2

FIGURE 13: Transient velocity contour inside the volute for the low flow-rate condition.

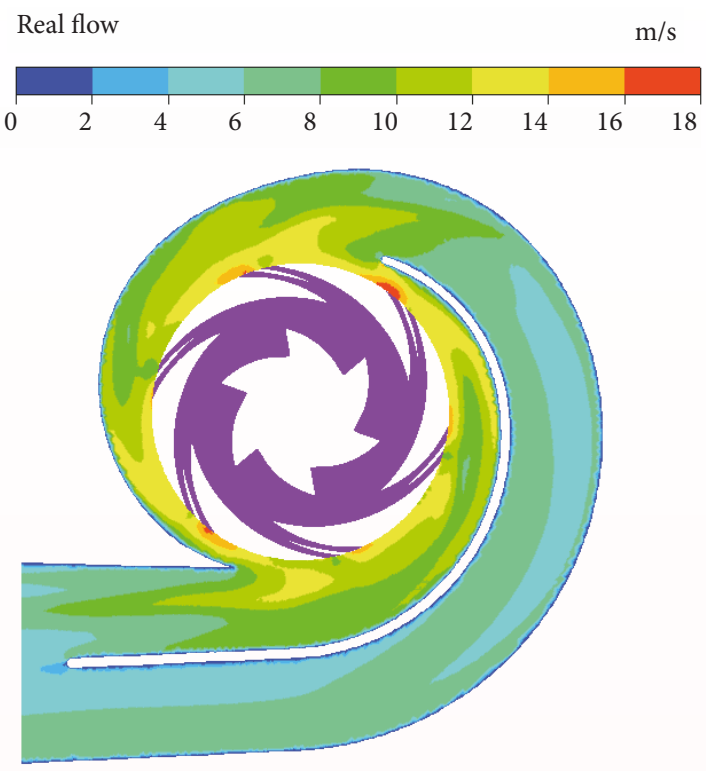

(a) Original flow field at $\mathrm{T} / 2$
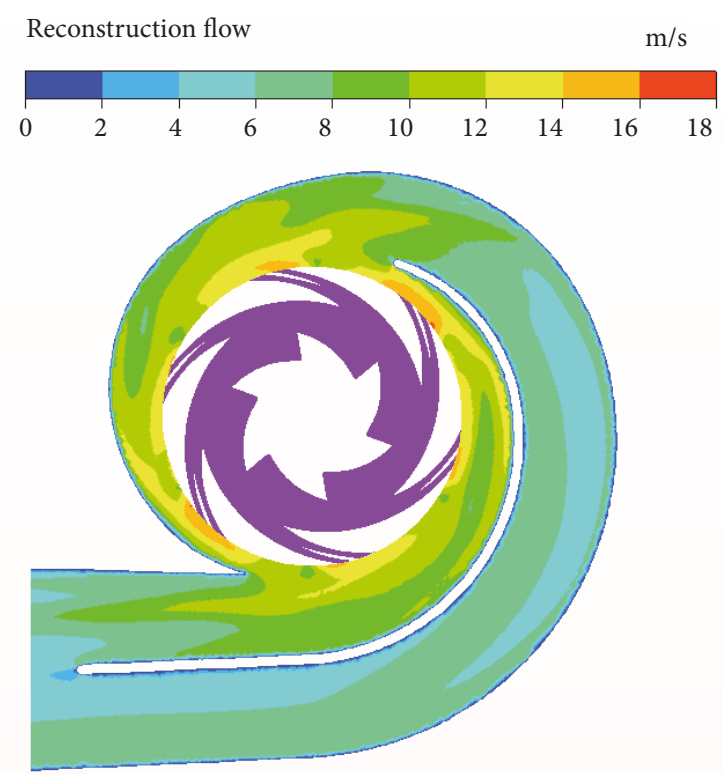

(b) Reconstructed flow field at $\mathrm{T} / 2$

FIGURE 14: Transient velocity contour inside the volute for the nominal flow-rate condition.

different times. It can be seen from the velocity contour that six high-speed fluid clusters are uniformly distributed in the circumferential direction of the volute inlet, and at these different times, the six high-speed fluid clusters always occupy a dominant position. The motion process of the six high-speed fluid clusters is consistent with the rotary scanning process of the blade, which indicates that the unsteady flow structure in the volute caused by rotor-stator interaction is not fixed but is synchronized with the rotation of the blade.

\section{Conclusions}

In this paper, the internal flow of the centrifugal pump is numerically simulated, and the velocity field of the midspan section of the centrifugal pump volute is analyzed by 
Reconstruction flow with second mode

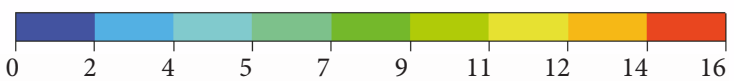

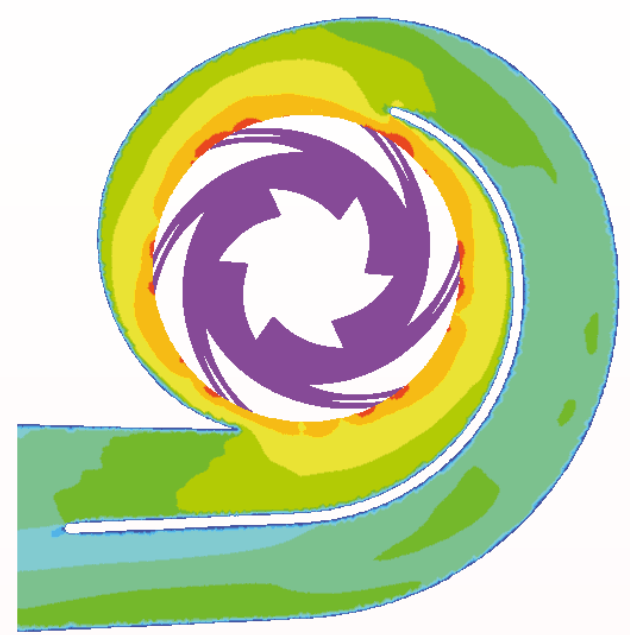

$\mathrm{T} / 4$

Reconstruction flow with second mode
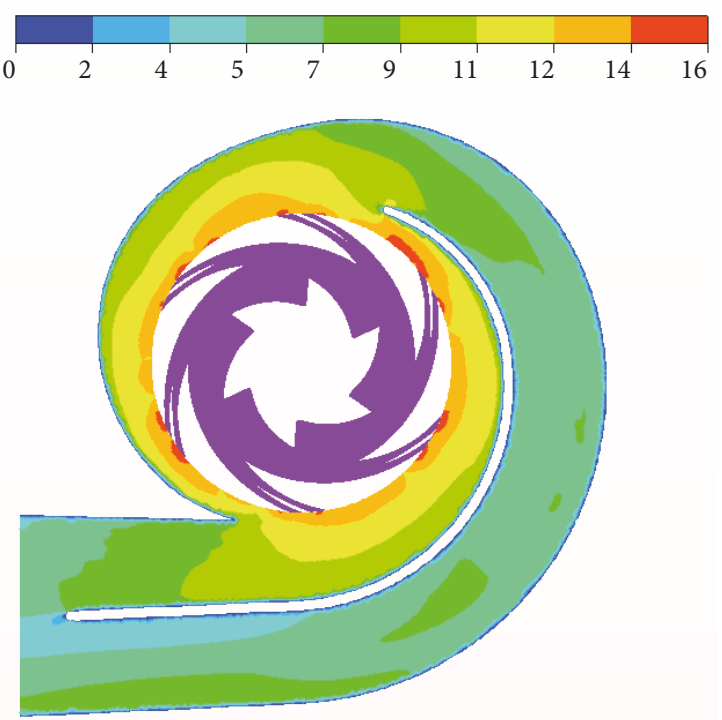

$3 \mathrm{~T} / 4$
Reconstruction flow with second mode $\mathrm{m} / \mathrm{s}$
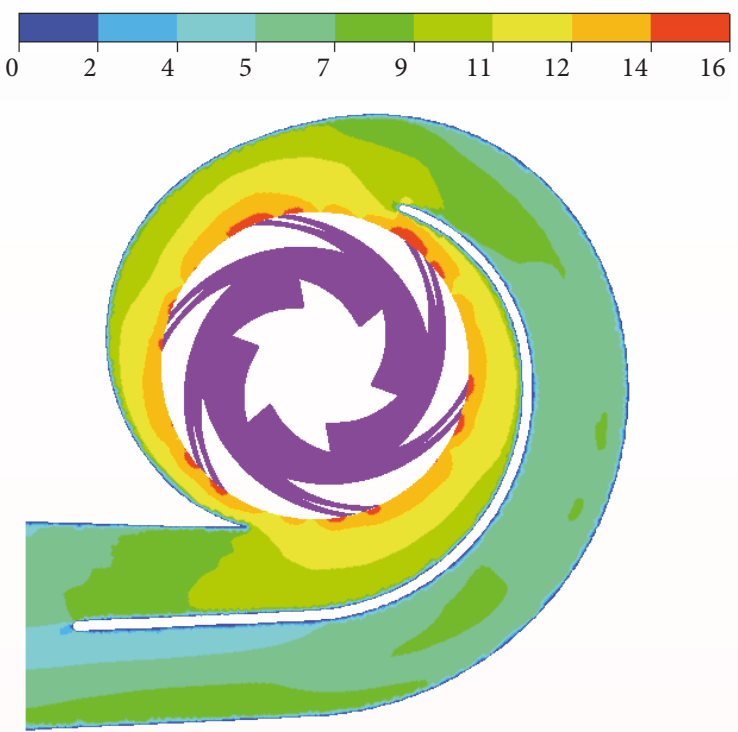

$\mathrm{T} / 2$

Reconstruction flow with second mode $\mathrm{m} / \mathrm{s}$
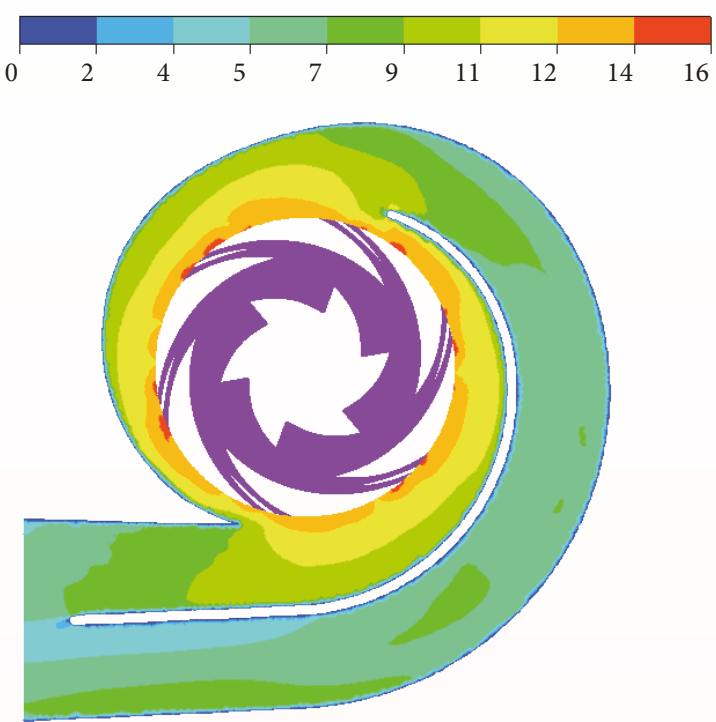

$\mathrm{T}$

FIGURE 15: Reconstructed velocity contour with second-order mode for the nominal flow-rate condition.

dynamic mode decomposition for the low flow-rate condition and the nominal flow-rate condition. The characteristic frequency of the flow field and the flow mode of different frequencies are extracted, and the reduced order analysis of the original flow field is realized. In the extracted flow modes of different frequencies, the first-order mode is the flow structures occupying the dominant position of the original flow field for the low flow-rate condition and the nominal flow-rate condition. The second- and third-order modes are the main oscillation modes of the original flow field, and the two modes' characteristic frequency is consistent with the blade passing frequency and $2 \mathrm{x}$ blade passing frequency obtained by FFT, which proves that the rotor-stator interaction between the impeller and the volute is the main reason for the unsteady flow in the volute. Next, comparing the mode velocity contours of each order for the condition of low flow rate and nominal flow rate, when the flow rate of the centrifugal pump is reduced, the existence of the tongue and the diaphragm will have a certain inhibitory effect on the unstable flow structure inside the volute, but it is more prone to occur in low frequency unsteady flow. Finally, the flow field of a single mode is reconstructed by superimposing 
the second-order mode to the first-order mode under the rated condition. And the results show that six high-speed fluid clusters dominated at the volute inlet rotate periodically with the blade. Generally speaking, the combination of DMD method and CFD unsteady calculation method is beneficial to understand the flow mechanism in the volute of centrifugal pump by extracting the coherent structure of unsteady flow.

\section{Nomenclature}

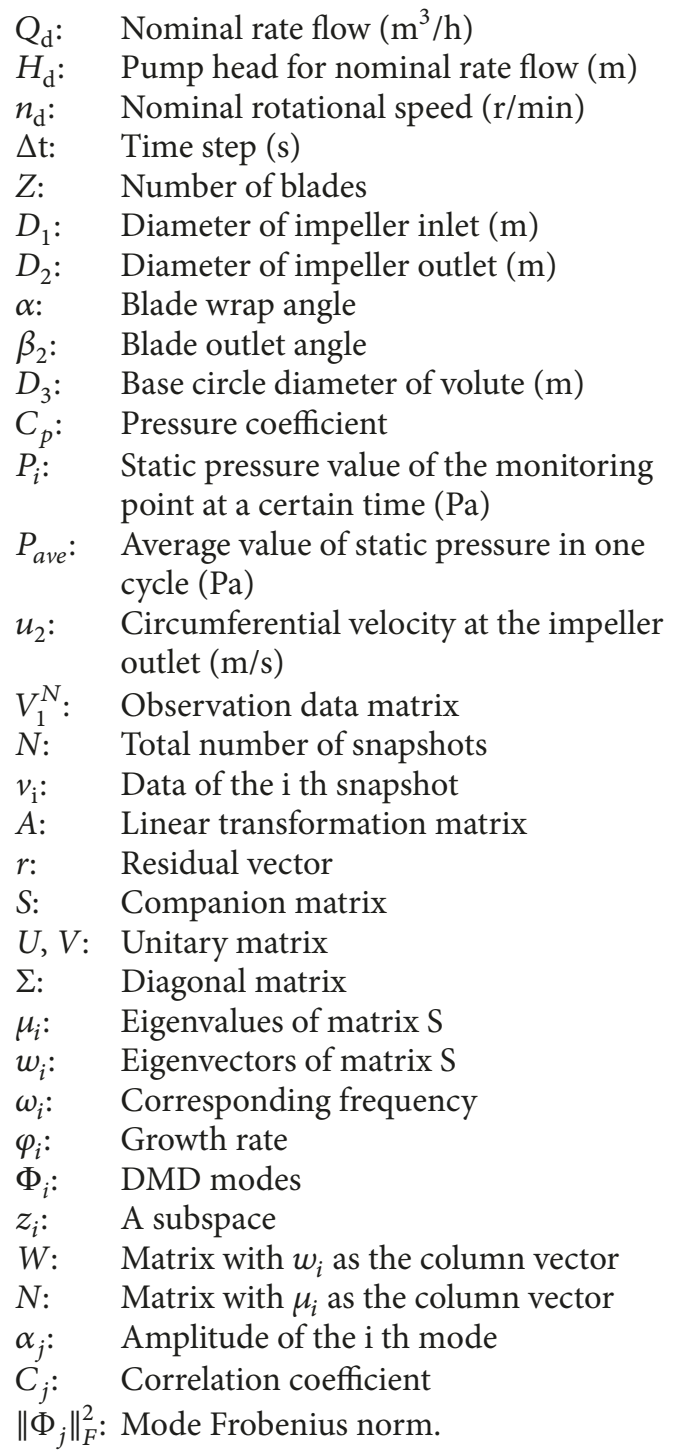

\section{Abbreviation}

DMD: Dynamic mode decomposition

FFT: Fast Fourier transformation

CFD: Computational fluid dynamics.

\section{Data Availability}

The data used to support the findings of this study are available from the corresponding author upon request.

\section{Conflicts of Interest}

The authors have approved the final version of the manuscript submitted. There is no financial or personal interest.

\section{Acknowledgments}

The authors are grateful to the financial support from the National Natural Science Foundation of China (Research Project No. 51866009).

\section{References}

[1] J. D. H. Kelder, R. J. H. Dijkers, B. P. M. Van Esch, and N. P. Kruyt, "Experimental and theoretical study of the flow in the volute of a low specific-speed pump," Fluid Dynamics Research, vol. 28, no. 4, pp. 267-280, 2001.

[2] J. González, J. Fernández, E. Blanco, and C. Santolaria, "Numerical simulation of the dynamic effects due to impeller-volute interaction in a centrifugal pump," Journal of Fluids Engineering, vol. 124, no. 2, pp. 348-355, 2002.

[3] J. Keller, E. Blanco, R. Barrio, and J. Parrondo, "PIV measurements of the unsteady flow structures in a volute centrifugal pump at a high flow rate," Experiments in Fluids, vol. 55, no. 10, p. 1820, 2014

[4] L. Tan, B. Zhu, Y. Wang, S. Cao, and S. Gui, "Numerical study on characteristics of unsteady flow in a centrifugal pump volute at partial load condition," Engineering Computations (Swansea, Wales), vol. 32, no. 6, pp. 1549-1566, 2015.

[5] H. Alemi, S. A. Nourbakhsh, M. Raisee, and A. F. Najafi, "Development of new "multivolute casing" geometries for radial force reduction in centrifugal pumps," Engineering Applications of Computational Fluid Mechanics, vol. 9, no. 1, pp. 1-11, 2015.

[6] F. J. Wang, L. X. Qu, L. Y. He, and J. Y. Gao, "Evaluation of flow-induced dynamic stress and vibration of volute casing for a large-scale double-suction centrifugal pump," Mathematical Problems in Engineering, vol. 2013, Article ID 764812, 9 pages, 2013.

[7] Y. Liu and L. Tan, "Tip clearance on pressure fluctuation intensity and vortex characteristic of a mixed flow pump as turbine at pump mode," Journal of Renewable Energy, vol. 129, pp. 606-615, 2018.

[8] Y. Hao and L. Tan, "Symmetrical and unsymmetrical tip clearances on cavitation performance and radial force of a mixed flow pump as turbine at pump mode," Journal of Renewable Energy, vol. 127, pp. 368-376, 2018.

[9] T. Lei, Y. Zhiyi, X. Yun, L. Yabin, and C. Shuliang, "Role of blade rotational angle on energy performance and pressure fluctuation of a mixed-flow pump," Proceedings of the Institution of Mechanical Engineers, Part A: Journal of Power and Energy, vol. 231, no. 3, pp. 227-238, 2017.

[10] T. Lei, X. Zhifeng, L. Yabin, H. Yue, and X. Yun, "Influence of T-shape tip clearance on performance of a mixed-flow pump," Proceedings of the Institution of Mechanical Engineers, Part A: Journal of Power and Energy, vol. 232, no. 4, pp. 386-396, 2018.

[11] P. J. Schmid and J. Sesterhenn, "Dynamic mode decomposition of numerical and experimental data," in Proceedings of the SixtyFirst Annual Meeting of the APS Division of Fliuid Mechanics, 2008.

[12] P. J. Schmid, "Dynamic mode decomposition of numerical and experimental data," Journal of Fluid Mechanics, vol. 656, pp. 5$28,2010$. 
[13] P. J. Schmid, L. Li, M. P. Juniper, and O. Pust, "Applications of the dynamic mode decomposition," Theoretical and Computational Fluid Dynamics, vol. 25, no. 1-4, pp. 249-259, 2011.

[14] A. Seena and H. J. Sung, "Dynamic mode decomposition of turbulent cavity flows for self-sustained oscillations," International Journal of Heat and Fluid Flow, vol. 32, no. 6, pp. 1098-1110, 2011.

[15] M. S. Hemati, M. O. Williams, and C. W. Rowley, "Dynamic mode decomposition for large and streaming datasets," Physics of Fluids, vol. 26, no. 11, 2014.

[16] J. H. Tu, C. W. Rowley, D. . Luchtenburg, S. L. Brunton, and J. N. Kutz, "On dynamic mode decomposition: theory and applications," Journal of Computational Dynamics, vol. 1, no. 2, pp. 391-421, 2014.

[17] C. Pan, D. Xue, and J. Wang, "On the accuracy of dynamic mode decomposition in estimating instability of wave packet," Experiments in Fluids, vol. 56, no. 8, p. 164, 2015.

[18] D. A. Bistrian and I. M. Navon, "The method of dynamic mode decomposition in shallow water and a swirling flow problem," International Journal for Numerical Methods in Fluids, vol. 83, no. 1, pp. 73-89, 2017.

[19] K. Taira, S. L. Brunton, S. T. Dawson et al., "Modal analysis of fluid flows: an overview," AIAA Journal, 2017.

[20] M. Liu, L. Tan, and S. Cao, "Dynamic mode decomposition of cavitating flow around ALE 15 hydrofoil," Journal of Renewable Energy, vol. 139, pp. 214-227, 2019.

[21] M. Liu, L. Tan, and S. Cao, "Dynamic mode decomposition of gas-liquid flow in a rotodynamic multiphase pump," Journal of Renewable Energy, vol. 139, pp. 1159-1175, 2019.

[22] J. Kou and W. Zhang, "An improved criterion to select dominant modes from dynamic mode decomposition," European Journal of Mechanics - B/Fluids, vol. 62, pp. 109-129, 2017. 


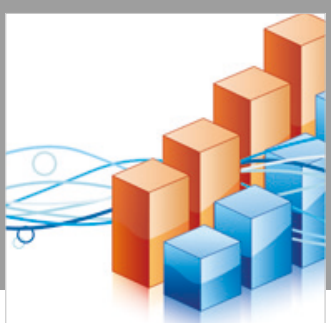

Advances in

Operations Research

\section{-n-m}
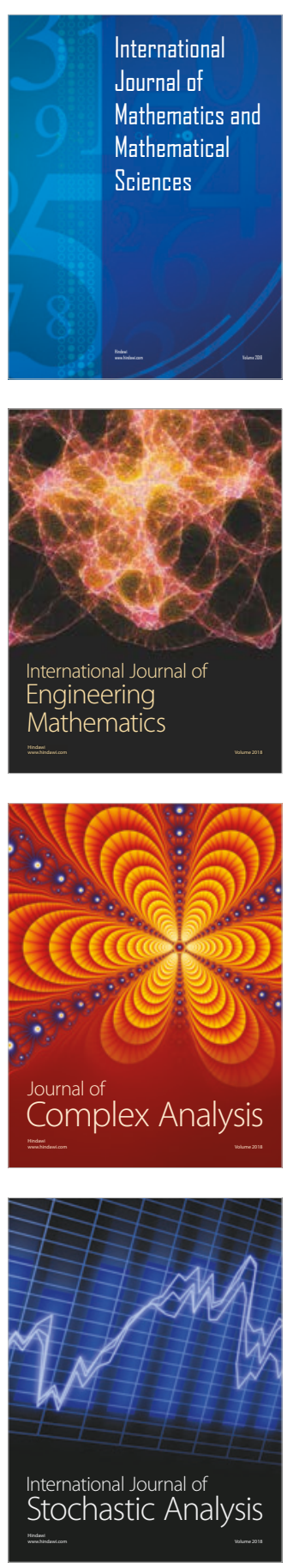
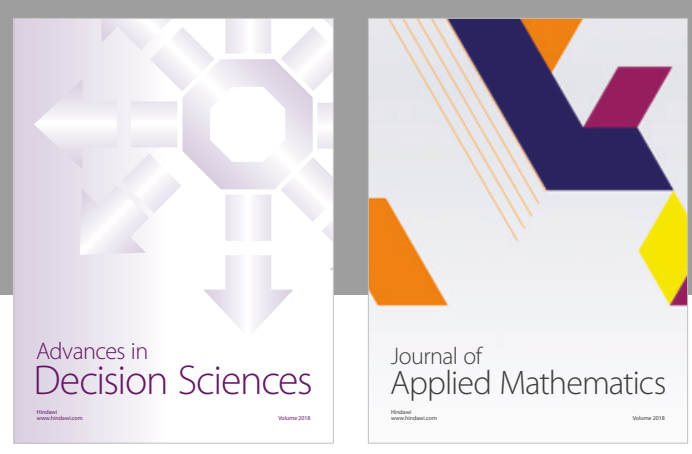

Journal of

Applied Mathematics
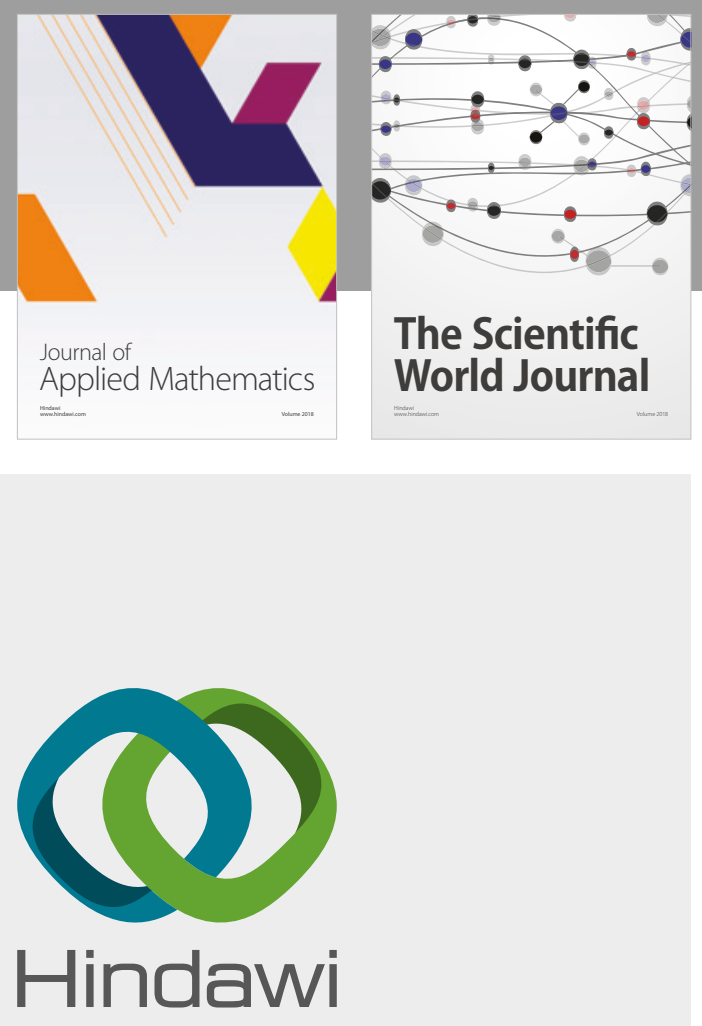

Submit your manuscripts at

www.hindawi.com

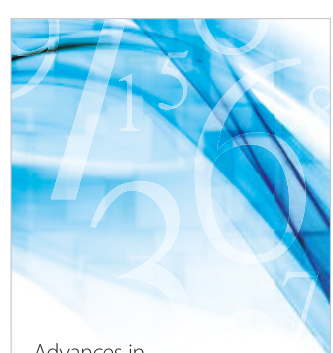

Advances in
Numerical Analysis
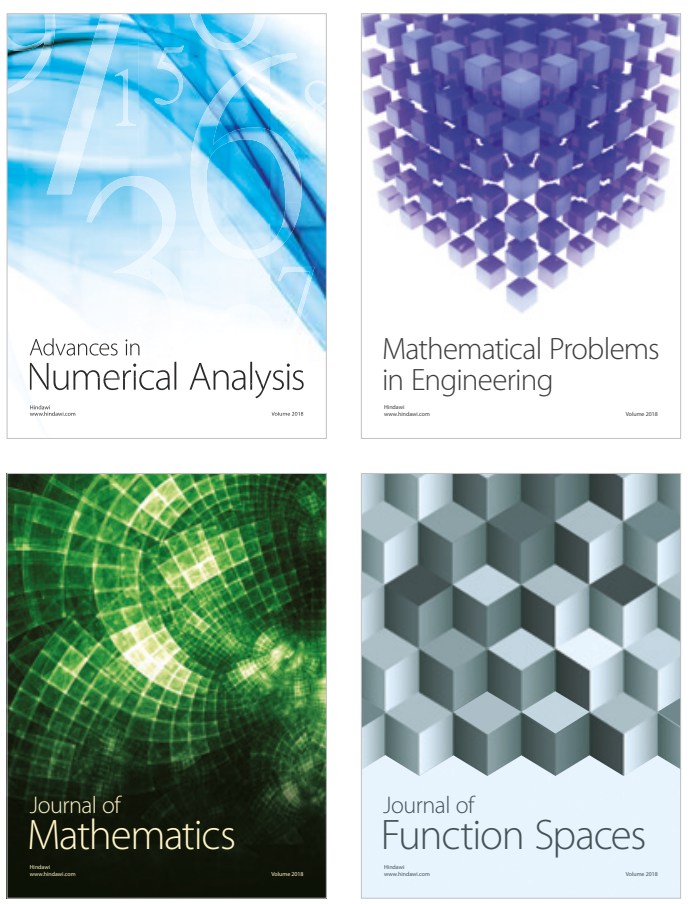

Mathematical Problems in Engineering

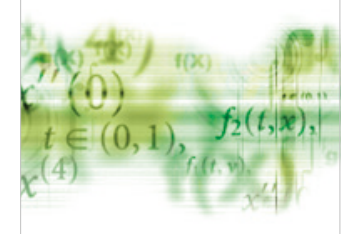

International Journal of

Differential Equations

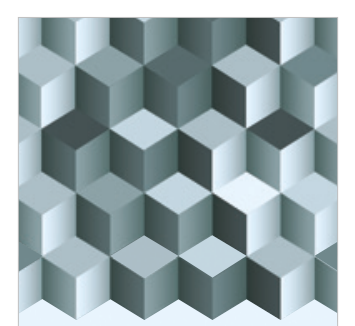

Journal of

Function Spaces

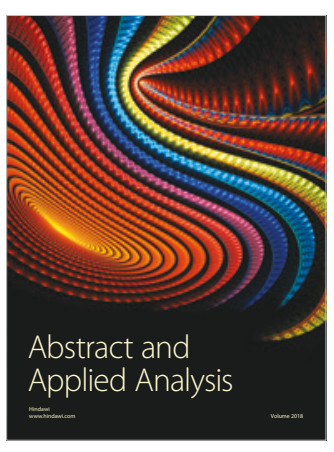

The Scientific

World Journal

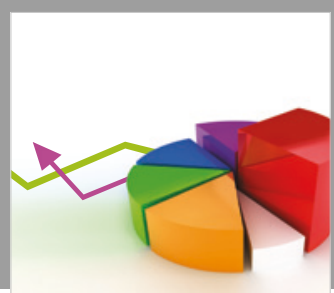

Journal of

Probability and Statistics
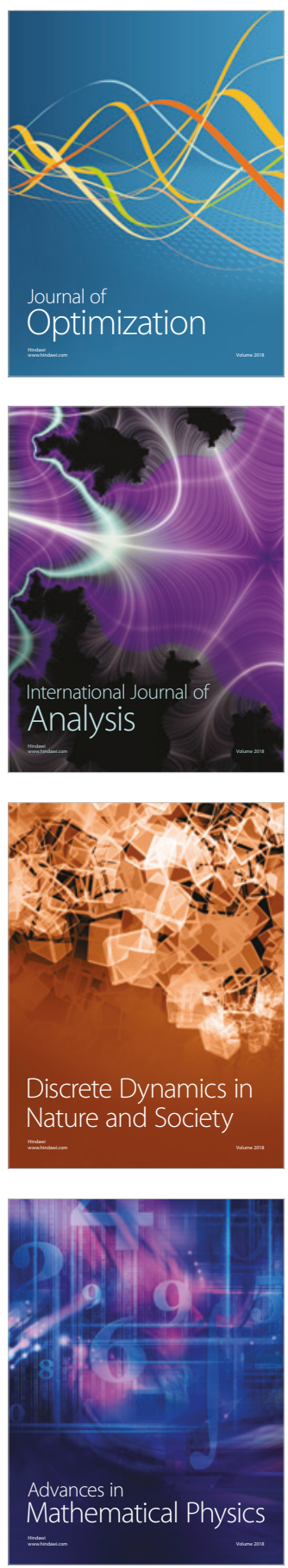